\title{
Disruption of EXOC6B in a patient with developmental delay, epilepsy, and a de novo balanced $\mathrm{t}(2 ; 8)$ translocation
}

\begin{abstract}
Anne Frühmesser ${ }^{1,6}$, Jonathon Blake ${ }^{2,6}$, Edda Haberlandt ${ }^{3}$, Bianka Baying ${ }^{2}$, Benjamin Raeder ${ }^{4}$, Heiko Runz ${ }^{5}$, Ana Spreiz ${ }^{1}$, Christine Fauth ${ }^{1}$, Vladimir Benes ${ }^{2}$, Gerd Utermann ${ }^{1}$, Johannes Zschocke ${ }^{1}$ and Dieter Kotzot ${ }^{\star, 1}$

Most balanced chromosomal aberrations are not associated with a clinical phenotype, however, in some patients they may disrupt gene structure. With the development of various next-generation sequencing techniques, fast and specific analyses of the breakpoint regions of chromosomal rearrangements are possible. Here, we report on a 19-year-old woman with a de novo balanced translocation $\mathrm{t}(2 ; 8)(\mathrm{p} 13.2 ; \mathrm{q} 22.1)$ and a severe clinical phenotype including intellectual disability, epilepsy, behavioral features resembling autism, and minor dysmorphic features. By next-generation sequencing, we defined the breakpoints and found disruption of the exocyst complex component $6 B$ (EXOC6B) gene in intron 1 on chromosome $2 \mathrm{p} 13.2$ involving two Alu elements with a homology of $81 \%$. No gene was found at the respective breakpoint on chromosome 8 . Expression analysis of the EXOC6B in blood lymphocytes and buccal smear revealed reduced expression in the patient in comparison with the control. Our findings in combination with one recently published case and one other patient listed in DECIPHER v5.1 indicate EXOC6B as a gene relevant for intellectual development and electrophysiological stability.
\end{abstract}

European Journal of Human Genetics (2013) 21, 1177-1180; doi:10.1038/ejhg.2013.18; published online 20 February 2013

Keywords: balanced translocation; gene disruption; next-generation sequencing

\section{INTRODUCTION}

De novo balanced reciprocal translocations can be found in $0.14 \%$ of all newborns. ${ }^{1}$ In most of these cases, the aberrations are not related to any clinical phenotype. However, some patients with apparently balanced translocations have an otherwise unexplained disease. In a few reports such conditions are explained by the presence of microdeletions or microduplications at the breakpoint regions. ${ }^{2}$ Also an interference of a gene or regulatory elements in the breakpoint region can cause the clinical phenotype. ${ }^{3,4}$ As a result of the low resolution of conventional chromosomal analysis and fluorescence in situ hybridization most of these aberrations have remained undetected and the phenotypic relevance of the translocation remains often unclear. Even high-resolution microarrays can only detect small deletions or duplications down to a size of approximately $10 \mathrm{~kb}$ and cannot characterize the breakpoints themselves. This may explain why so far, research and characterization of chromosomal breakpoints have only been done in some cases of well-known microdeletions and microduplications. The development of various next-generation sequencing techniques now offers a method to analyze such aberrations. With these techniques it is possible to characterize breakpoints at the base pair level, to analyze the whole genome for other small structural aberrations, and therefore to understand the underlying chromosomal disorders and mechanisms in patients with a clinical phenotype and an apparently balanced aberration.
Here, we report on a 19-year-old woman with a de novo balanced translocation $\mathrm{t}(2 ; 8)(\mathrm{p} 13.2 ; \mathrm{q} 22.1)$, global developmental delay, epilepsy, and minor dysmorphic features.

\section{CLINICAL REPORT}

The patient is the first child of a 27 -year-old mother and her unrelated 37-year-old husband. One previous pregnancy ended in an abortion in the ninth week. Three younger siblings are healthy. Family history was unremarkable. Apart from low maternal blood pressure, pregnancy was also unremarkable. Spontaneous birth was at 38 weeks of gestation. Length $(51 \mathrm{~cm})$, weight $(3590 \mathrm{~g})$, and occipitofrontal head circumference $(\mathrm{OFC} ; 36 \mathrm{~cm}$ ) were above the 90 th centile. At the age of 6 months, infantile spasms occurred and a global developmental delay was recognized. Electroencephalography detected hypsarrhythmia and anticonvulsive therapy with phenobarbital and vigabatrin was started. Infantile spasms were ceased but tonic seizures and generalized epileptic activity were observed. The patient was seizure free with the drug regime of vaproic acid, lamotrigine, ethosuximide, and clobazame at the age of 4 years. EEG was normal since this age and drug medication could be stopped. Parents described developmental delay at the age of 8 months, severe behavioral disorders with autistic and aggressive features, and stereotypical movement disorder. Walking was possible at the age of 22 months.

\footnotetext{
${ }^{1}$ Division of Human Genetics, Department of Medical Genetics, Molecular and Clinical Pharmacology, Innsbruck Medical University, Innsbruck, Austria; ${ }^{2}$ European Molecular Biology Laboratory (EMBL), Genomics Core Facility, Heidelberg, Germany; ${ }^{3}$ Clinical Department of Pediatrics I, Innsbruck Medical University, Innsbruck, Austria; ${ }^{4}$ European Molecular Biology Laboratory (EMBL), Korbel Lab, Heidelberg, Germany; ${ }^{5}$ Institute of Human Genetics, University of Heidelberg, Heidelberg, Germany

${ }^{6}$ These author contributed equally to this work.

*Correspondence: Dr D Kotzot, Division of Human Genetics, Department of Medical Genetics, Molecular and Clinical Pharmacology, Schoepfstr. 41, Innsbruck A-6020, Austria. Tel: +0043 512 9003-70531; Fax: +0043 512 9003-73510; E-mail: DieterKotzot@gmx.de

Received 13 October 2012; revised 20 December 2012; accepted 17 January 2013; published online 20 February 2013
} 
At the age of 11 years, height was $142 \mathrm{~cm}$ (50th centile), weight was $38.9 \mathrm{~kg}$ (50th-75th centile), and OFC was $50.5 \mathrm{~cm}$ (3rd-10th centile). Dysmorphic features included temporal narrowing, strabismus, a broad nasal bridge, a slight X-position of the arms, tapering finger, short fifth fingers, and a large distance between the first and the second toes at both feet (Figures 1a-f).

At the age of 19 years, height was $162 \mathrm{~cm}$ (25th centile), weight $58 \mathrm{~kg}$ (50th-75th centile), and OFC $54 \mathrm{~cm}$ (25th-50th centile). Speech was reduced to a few words and features resembling autism remained. Menarche was at the age of 16 years. Dysmorphic features included strabismus, a thin upper lip, and slightly posteriorly rotated ears (Figures 1g-i). Ear length was $6.5 \mathrm{~cm}$ (75th-90th centile), inner canthal distance and outer canthal distance were $2.5 \mathrm{~cm}$ (3rd-25th centile), and $8.5 \mathrm{~cm}$ (50th centile), respectively. Total hand length and middle finger length were $17 \mathrm{~cm}$ (50th centile) and $7.3 \mathrm{~cm}$ (50th-75th centile).

\section{METHODS AND RESULTS}

Investigation of our patient by conventional karyotype analysis revealed a balanced translocation of the short arm of chromosome 2 and the long arm of chromosome 8 (Figure 2a). Both parents had normal karyotypes. The initial karyotype of the patient was therefore written as $46, \mathrm{XX}, \mathrm{t}(2 ; 8)(\mathrm{p} 13 \sim 15 ; \mathrm{q} 22) d n$. SNP array investigation with the Illumina 1M-Duo Bead Chip (>1000 000 SNPs) according to the manufacturer's instructions was unremarkable. By microdissection of the derivative chromosomes from metaphases followed by microsatellite-mediated haplotype analysis, the translocation was shown to be of maternal origin. ${ }^{5}$ With next-generation sequencing by the Illumina Mate
Pair Library Prep Kit (Illumina Inc., San Diego, CA, USA) according to the manufacturer's instructions and subsequent analysis by the HiSeq2000 Sequencer (Illumina Inc.) the breakpoints of the balanced translocation could be narrowed down to a region between $73022374 \mathrm{bp}$ and 73025543 bp on 2p13.2 and 93711398 bp and 93712952 bp on 8q22.1 (hg build 37.3). Junction fragment PCR with the Phusion High-Fidelity DNA Polymerase (Finnzymes, Espoo, Finland) and subsequent Sanger sequencing revealed the breakpoints in an Alu element (AluSq2) on chromosome 8 between positions $93710009 \mathrm{bp}$ and $93710215 \mathrm{bp}$ (206 bp) and another Alu element (AluSx1) on chromosome 2 between positions $73023379 \mathrm{bp}$ and $73023582 \mathrm{bp}$ (203 bp) (hg build 37.3). Both elements are located in direct orientation and share a sequence homology of $81 \%$. The breakpoint on chromosome 2 is located in intron 1 of the exocyst complex component $6 B$ $(E X O C 6 B)$ gene whereas the breakpoint on chromosome 8 is not located in a coding sequence. Sanger sequencing analysis of all 22 exons of the EXOC6B gene, to exclude an autosomal recessive cause revealed no mutations. Expression analysis of EXOC6B by qRT-PCR with the Maxima SYBR Green/ ROX qPCR Master Mix (2X) (Fermentas, St Leon-Rot, Germany) according to the manufacturer's instructions in blood lymphocytes showed reduced expression to approximately $50 \%$ in the patient in comparison with the control (Figure 2b). As a result of the small amount of buccal smear obtained from the patient, an expression analysis of $E X O C 6 B$ in this tissue was only possible twice. Despite the high standard deviation, a reduction of the expression level can be clearly seen in the patient in comparison with the control (Figure 2b).

\section{DISCUSSION}

Generally, balanced translocations are not associated with a phenotype as there is no loss or gain of genetic material. Nevertheless, in

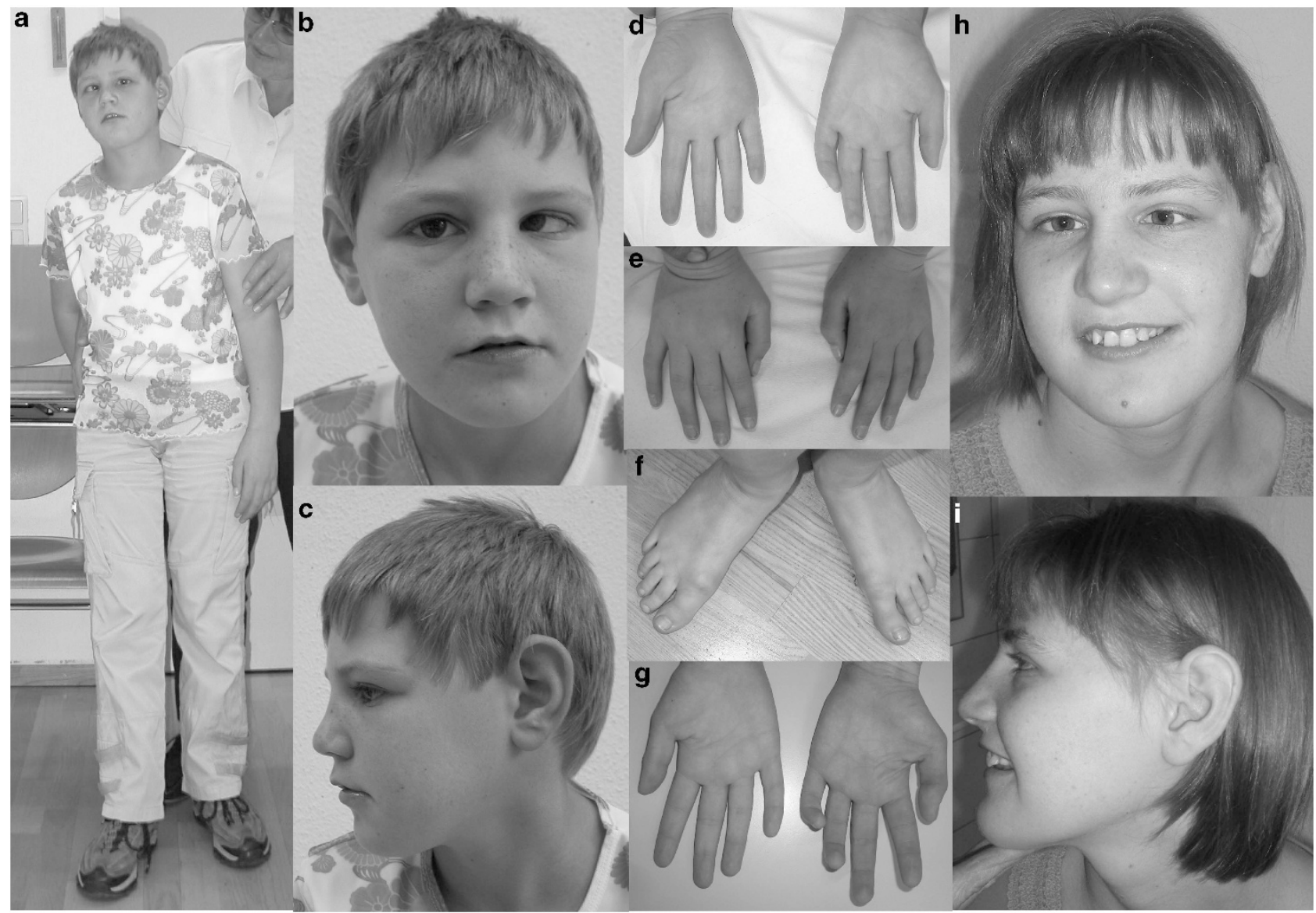

Figure 1 Patient at the age of 11 years $(\mathbf{a}-\mathbf{f})$ and at the age of 19 years (g-i). Dysmorphic features included temporal narrowing, strabismus, a broad nasal bridge, tapering finger, and short fifth fingers. 
a

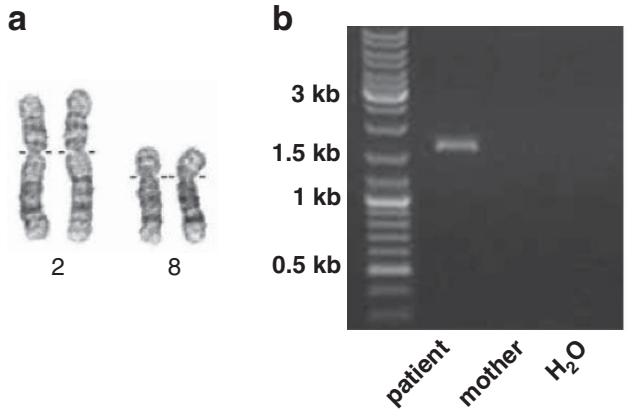

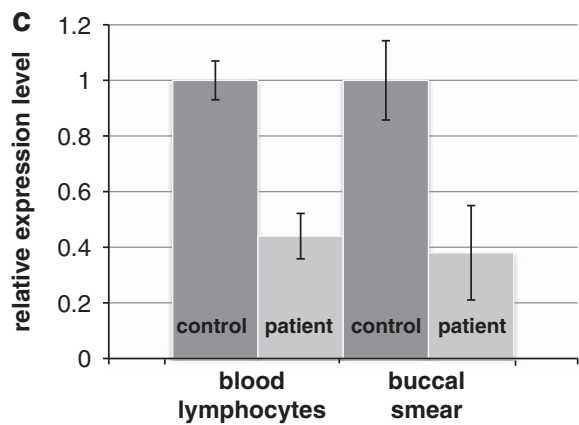

Figure 2 (a) Partial GTG karyogram of the patient showing chromosomes 2 and 8. (b) Result of the junction fragment PCR of the derivative chromosome 2 (forward primer is located on chromosome 8 and reverse primer is located on chromosome 2) in the patient and the mother as a negative control person. Only the patient shows a specific PCR product of approximately $1.6 \mathrm{~kb}$. In the control, no product can be detected. (c) Results of qRT-PCR analysis showing reduction of the expression level of EXOC6B in blood lymphocytes and buccal smear in our patient in comparison with the control.

some cases severe clinical phenotypes are described in the literature. ${ }^{6}$ Here, we report on a female patient with global developmental delay, epilepsy, minor dysmorphic features, and a de novo balanced translocation $\mathrm{t}(2 ; 8)(\mathrm{p} 13.2 ; \mathrm{q} 22.1)$. First, a microduplication or microdeletion was excluded by conventional chromosomal analysis and high-resolution SNP-array analysis. Then, by a specific nextgeneration sequencing approach the breakpoints of the translocation could be defined and EXOC6B was found to be interrupted by the breakpoint on chromosome 2p13.2. EXOC6B, also known as Sec15B and $\operatorname{Sec} 15 \mathrm{~L} 2$, is nearly ubiquitously expressed and spans $650 \mathrm{~kb}$. Little is known about the function of the gene. In 1996, TerBush et al ${ }^{7}$ identified the Saccharomyces cerevisiae homolog of EXOC6B as a component of the exocyst, a octameric protein complex. This complex mediates the tethering of post-Golgi secretory vesicles to the plasma membrane before exocytic fusion. ${ }^{8}$ Further publications showed interactions between the exocyst and multiple Ras-related small GTPases, including those that regulate the vesicular trafficking such as the Rab proteins as well as the actin cytoskeleton like the Rho family. ${ }^{9,10}$ In yeast, Wu et $a l^{11}$ reported a function of SEC15 in targeting transport vesicles to the budding site and assumed the protein to recruit other proteins in the exocyst complex. In humans, two Sec15 genes were identified, namely EXOC6 and EXOC6B that share $75 \%$ amino-acid identity but only $20 \%$ identity with Sec15 in yeast. ${ }^{12}$ Differences between the two human genes are located in the $\mathrm{N}$ - and C-terminus. In mammalians, only one study describes a mutation in the EXOC6 gene in mice that impairs erythroid iron assimilation by alteration of the recycling of the transferrin cycle endosomes and release of transferrin receptor exocytic vesicles. ${ }^{13}$ In Drosophila, loss of the Sec15 homolog is associated with a targeting defect of photoreceptors that coincides with mislocalization of specific cell adhesion and signaling molecules. ${ }^{14}$ Zhang et $a l^{10}$ further investigated the localization of Sec15 in Chinese hamster ovary cells. They found the protein associated with clusters of tubular/vesicular elements concentrated in the perinuclear region.

In 2008, Borsani et al ${ }^{12}$ reported a 4 -year-old male patient with left side renal agenesis, renal insufficiency, neutropenia, recurrent pulmonary infections, long bone diaphysis broadening, behavioral difficulties, growths retardation, ID, and a de novo $\mathrm{t}(2 ; 7)(\mathrm{p} 13 ; \mathrm{p} 12)$ balanced translocation involving the tensin3 (TNS3) gene on $7 \mathrm{p}$ and the EXOC6B gene on 2p. The breakpoint in the EXOC6B gene was located within the first intron. They detected an EXOC6B-TNS3 fusion transcript together with a TNS3-EXOC6B chimeric RNA in the patient's fibroblasts. The authors hypothesized that the phenotype of the patient results from a haploinsufficiency of one or both affected genes, a dominant-negative effect caused by the chimeric transcript, and/or an abnormal regulation in the expression of other genes next to the breakpoint.

A search in the DECIPHER database v5.1 (http://decipher.sanger. ac.uk) revealed one more patient (249203) with a 780-kb deletion including the whole EXOC6B gene as well as the proximally located CYP26B1 gene that is responsible for autosomal-recessively inherited craniosynostosis with radiohumeral fusions and other skeletal and craniofacial anomalies. ${ }^{15}$ The clinical phenotype includes ID, autistic features, as well as prominent ears. DECIPHER v5.1 predicts a haploinsufficiency score of $17 \%$ for EXOC6B. According to the definition of the haploinsufficiency score, this means that haploinsufficiency of this gene is most likely clinically relevant.

Our findings in combination with the available evidence indicate that the phenotype in our patient may be linked to reduced expression of EXOC6B. Considering that both our patient and the patient described in the DECIPHER database show autistic traits and the 4-year-old patient reported by Borsani et al ${ }^{12}$ was reported to have abnormal behavior, EXOC6B gene might also be a candidate gene for autistic behavior.

\section{CONFLICT OF INTEREST}

The authors declare no conflict of interest.

\section{ACKNOWLEDGEMENTS}

We thank the family for the excellent cooperation. This work was founded by a TWF-grant (D-151740-020-011) to AS.

1 Gardner RJM, Sutherland GR, Shaffer LG: Chromosome Abnormalities and Genetic Counseling. Oxford University Press, 2012.

2 Gonzalez-Gonzalez C, Garcia-Hoyos M, Hernaez Calzon R et al: Microdeletion found by array-CGH in girl with Blepharophimosis syndrome and apparently balanced translocation t(3;15)(q23;q25). Ophthalmic Genet 2011; 33: 107-110.

3 Backx L, Seuntjens E, Devriendt K, Vermeesch J, Van Esch H: A balanced translocation $\mathrm{t}(6 ; 14)(\mathrm{q} 25.3 ; \mathrm{q} 13.2)$ leading to reciprocal fusion transcripts in a patient with intellectual disability and agenesis of corpus callosum. Cytogenet Genome Res 2011; 132: 135-143.

4 De Marco P, Raso A, Beri $\mathrm{S}$ et al: A de novo balanced translocation $\mathrm{t}(7 ; 12)(\mathrm{p} 21.2 ; \mathrm{p} 12.3)$ in a patient with Saethre-Chotzen-like phenotype downregulates TWIST and an osteoclastic protein-tyrosine phosphatase, PTP-oc. Eur J Med Genet 2011; 54: e478-e483.

5 Höckner M, Spreiz A, Frühmesser AO et al: Parental origin of de novo cytogenetically balanced reciprocal non-robertsonian translocations. Cytogenet Genome Res 2012; 136: 242-245. 
6 Kurosawa K, Tanoshima-Takei M, Yamamoto $\mathrm{T}$ et al: Sirenomelia with a de novo balanced translocation 46,X,t(X;16)(p11.23;p12.3). Congenit Anom (Kyoto) 2012; 52: 106-110.

7 TerBush DR, Maurice T, Roth D, Novick P: The Exocyst is a multiprotein complex required for exocytosis in Saccharomyces cerevisiae. EMBO J 1996; 15 6483-6494.

$8 \mathrm{He}$ B, Guo W: The exocyst complex in polarized exocytosis. Curr Opin Cell Biol 2009; 21: $537-542$.

9 Brymora A, Valova VA, Larsen MR, Roufogalis BD, Robinson PJ: The brain exocyst complex interacts with RalA in a GTP-dependent manner: identification of a novel mammalian Sec3 gene and a second Sec15 gene. J Biol Chem 2001; 276: 29792-29797.

10 Zhang XM, Ellis S, Sriratana A, Mitchell CA, Rowe T: Sec15 is an effector for the Rab11 GTPase in mammalian cells. J Biol Chem 2004; 279: 43027-43034.
11 Wu S, Mehta SQ, Pichaud F, Bellen HJ, Quiocho FA: Sec15 interacts with Rab11 via a novel domain and affects Rab11 localization in vivo. Nat Struct Mol Biol 2005; 12 879-885.

12 Borsani G, Piovani G, Zoppi N et al: Cytogenetic and molecular characterization of a de-novo $t(2 p ; 7 p)$ translocation involving TNS3 and EXOC6B genes in a boy with a complex syndromic phenotype. Eur J Med Genet 2008; 51: 292-302.

$13 \mathrm{Lim} \mathrm{JE}$, Jin O, Bennett $\mathrm{C}$ et al: A mutation in Sec15/1 causes anemia in hemoglobin deficit (hbd) mice. Nat Genet 2005; 37: 1270-1273.

14 Mehta SQ, Hiesinger PR, Beronja S et al: Mutations in Drosophila sec15 reveal a function in neuronal targeting for a subset of exocyst components. Neuron 2005; 46: 219-232.

15 Laue K, Pogoda HM, Daniel PB et al: Craniosynostosis and multiple skeletal anomalies in humans and zebrafish result from a defect in the localized degradation of retinoic acid. Am J Hum Genet 2011; 89: 595-606. 